\title{
TRADUCCIÓN ENTRE ESPAÑOL ESCRITO Y LENGUA DE SEÑAS URUGUAYA VIDEOGRABADA: UN NUEVO DESAFÍO
}

\author{
Leonardo Peluso* \\ Universidad de la República
}

\begin{abstract}
Resumen: El presente trabajo trata del concepto de textualidad diferida que es más abarcativo que el de escritura, dado que permite incluir a las videograbaciones en lengua de señas. $\mathrm{Si}$ se considera que las videograbaciones en lengua de señas son textualidad diferida, se puede sostener, también, que se desarrolla una cultura letrada en torno a éstas (entendida como aquella cultura que se genera a partir de la masificación del uso de una textualidad diferida, con sus prácticas sociales e institucionales concomitantes). A partir de esta idea, mostraré que es posible la traducción entre lenguas orales escritas y lenguas de señas videograbadas. También señalaré que, para el caso de Uruguay y su lengua de señas (la LSU), este tipo de traducción es una necesidad imperiosa ante una comunidad sorda cada vez más exigente, que ocupa nuevos espacios sociales.
\end{abstract}

Palabras clave: Textualidad diferida. Traducción. Videograbaciones. Escritura.

\footnotetext{
* Leonardo Peluso. Licenciado en Psicología y en Lingüística (Universidad de la República, Uruguay). Magister en Ciencias Humanas (Universidad de la República, Uruguay) y Doctor en Letras (Universidad Nacional de Córdoba, Argentina). Profesor Agregado en la Universidad de la República (Uruguay) donde ejerzo el cargo de coordinador de la Tecnicatura en Interpretación LSU-español (Facultad de Humanidades y Ciencias de la Educación) y la Especialización en Inclusión Social y Educativa (Facultad de Psicología). Montevideo, Salto y Tacuarembó; Uruguay. Email: leonardo@psico.edu.uy
} 


\title{
TRANSLATION BETWEEN WRITTEN SPANISH AND VIDEO-RECORDED URUGUAYAN SIGN LANGUAGE: A NEW CHALLENGE
}

\begin{abstract}
This paper deals with the concept of deferred textuality which is more comprehensive than writing, as it allows the inclusion of sign language video-recordings. If one considers that sign language videorecordings are deferred textuality, it can also be argued that a literate culture can be developed around them, understood as the culture built around deferred textuality and the social and institutional practices they spawn. From this idea, I will show that the translation between oral languages written texts and sign languages video-recorded texts is also possible. I will also point out that, in the case of Uruguay and its sign language (LSU), this kind or translation is imperative in front of an increasingly demanding Deaf Community, occupying new social spaces. Keywords: Deferred textuality. Tanslation. Video-recordings. Writing.
\end{abstract}

\section{Introducción}

La tecnología de videograbación de la lengua de señas (LSU para el caso de Uruguay) puede volverse una tecnología especialmente útil, para los sordos, en la conformación de una textualidad diferida, teniendo en cuenta que la LSU no cuenta con una tecnología de escritura y manteniendo ciertas especificidades en su producción.

Es muy importante resaltar que, cuando hablo de videograbaciones como una forma alternativa a la escritura, hago referencia a una especialización que está ocurriendo en las estructuras y funciones de la grabación en lengua de señas; que se diferencian en forma y función del conjunto general de productos grabados, tales como películas, documentales, etcétera. Son un tipo de grabación en el que el centro absoluto es el texto lingüístico y su finalidad es la de funcionar como texto diferido, con similar estructura y función descontextualizada que un texto escrito. Son videos en los que queda privilegiado el hablante diciendo su texto.

En este marco me parece clave distinguir entre prácticas de interpretación y prácticas de traducción, en la medida en que involu- 
cran actividades y contextos bien diferenciados, así como también me parece imprescindible que se desarrollen y consoliden equipos traductológicos que puedan comenzar a generar un corpus traducido de la LSU.

En este trabajo mostraré los resultados de mi tesis de doctorado, realizada en la Universidad Nacional de Córdoba, y de dos línea de investigación que se llevan adelante en la Tecnicatura Universitaria de Interpretación LSU-español (TUILSU), de la Facultad de Humanidades y Ciencias de la Educación de la Universidad de la República, Uruguay, en las que, por un lado, se está investigando sobre los alcances de las videograbaciones para la realización de una textualidad diferida en LSU y, por otro lado, se está trabajando en los procesos traductológicos que involucran al español escrito y a la LSU videograbada.

\section{Características generales de la textualidad diferida: la es- critura y la videograbación}

En la literatura general que trata las relaciones entre oralidad y escritura se tienden a confundir escritura con textualidad diferida, generando ciertos problemas a la hora de distinguir entre ambas modalidades de textualidad y a la hora de separar, por un lado, a las tecnologías que se aplican a las lenguas y, por otro lado, a las propias lenguas a las que se aplican dichas tecnologías. Teniendo en cuenta esto, en este trabajo prefiero llamar textualidad diferida (y no escritura) a todos aquellos textos que son apartados del momento de enunciación por medio de alguna tecnología de la lengua, sea esta tecnología un sistema de escritura o una herramienta de registro (audio o video grabaciones). Por su parte, mantendré el término cultura letrada para hacer referencia a las culturas que son afectadas por las prácticas discursivas habilitadas por el desarrollo y generalización de una textualidad diferida, tanto escrita como videograbada.

Las características que clásicamente fueron atribuidas a los textos escritos pueden ser fácilmente generalizables para los textos 
diferidos. Según Ong (1982), Sampson (1996) y Goody y Watt (1996) las propiedades esenciales de los textos escritos son la permanencia y la objetivación.

Por permanencia se entiende que los textos escritos, por ser tecnologizados, pueden permanecer más allá del momento de enunciación, habiendo perdido esa característica de la oralidad que Ong (1982) denominó evanescencia. De esta forma, los textos diferidos se separan de las coordenadas físicas de su enunciación, para perdurar más allá de estas.

Por objetivación estos autores entienden que el efecto que tiene aplicar una tecnología sobre la lengua es convertir al texto en un objeto. Por la aplicación de la tecnología, el texto se vuelve un objeto material que es, por lo tanto, manipulable. Dado un texto vuelto objeto, este se puede rayar, dibujar, cortar, desarmar, editar (para el caso de videos), o cualquier operación física que se quiera realizar. Este tipo de manipulación es imposible de realizar en la textualidad oral, dado su carácter evanescente.

En la medida en que los textos diferidos son objetos permanentes y materiales, adquieren otras propiedades relacionadas con su manipulación: la interpretabilidad, una particular forma de actividad metalingüística, la planificación y el control y la posibilidad de archivo.

Por interpretabilidad, se entiende la actividad lingüística y discursiva vinculada a la producción y a la comprensión de textos que están fuera del momento de enunciación, en donde escritor y lector no se tienen, de forma directa, para negociar los significados del decir y de lo dicho (ONG, 1982; OLSON, 1996; GOODY y WATT, 1996). El lector debe interpretar el texto en independencia de su interacción con quien lo haya escrito, y lo mismo le ocurre al escritor.

Por desarrollo de una particular actividad metalingüística se entiende, siguiendo a Olson (1996), que las culturas letradas conllevan el surgimiento de lo que este autor denominó metalenguaje oral, que es la actividad metalingüística asociada al manejo de una textualidad objetivada. Esta actividad metalingüística utiliza categorías de análisis para entender las diferentes unidades que confor- 
man un texto: fonemas, morfemas, palabras, oraciones, etcétera. A través de estas categorías analíticas los sujetos letrados, aquellos que manejan la textualidad diferida, piensan y actúan sobre los textos, lo que hace al desarrollo de formas de procesamiento del lenguaje diferentes a las que existen en la oralidad.

Por archivo se entiende la posibilidad física de hacer acopio de los textos diferidos. Esto requiere de la especialización de diferentes soportes de mantenimiento y clasificación de un número siempre creciente de textos diferidos, así como de instituciones que guardan y utilizan dichos textos.

Asimismo, y como efecto de la permanencia y de la objetivación, el texto diferido se vuelve planificado y controlado. El texto se transforma en un objeto que queda por fuera del momento de su enunciación y por lo tanto, como ya señalé, requiere de un procesamiento específico y diferente del que ocurre cuando se tiene al interlocutor en frente y se co-construye con él los significados. Este procesamiento incluye un fuerte componente de planificación y de control metalingüístico (OCHS, 1979, OLSON, 1966). Quien escribe o lee puede interponer una estructura de demora que, mediante las categorías metalingüísticas analíticas, le permite pensar detenidamente sobre el texto, tomar decisiones, controlar las estructuras y planificar lo que se desea escribir.

Teniendo esta definición general de textualidad diferida, es posible sostener que en la actualidad existen dos grandes tecnologías que habilitan dicha textualidad: los sistemas de escritura y las audio y videograbaciones. A estas tecnologías, que estan en la base de la textualidad diferida, se las las ha denominado tecnologías de la lengua (PELUSO, 2007; LARRINAGA; PELUSO, 2009; PELUSO; SILVEIRA, 2012).

Las videograbaciones comparten con la escritura esta propiedad de permanencia, dado que el texto videograbado también queda fijado; y también comparten la propiedad de objetivación, dado que materializan al texto y lo vuelven un objeto manipulable externo al hablante.

De esta forma, se puede sostener que los textos escritos y los textos videograbados comparten muchas propiedades, lo que hace 
posible que, bajo ciertas condiciones de producción, las videograbaciones en lengua de señas puedan tomar las estructuras y funciones que en nuestras culturas letradas son particulares de la escritura (PELUSO, 2007; LARRINAGA; PELUSO, 2009; PELUSO; SILVEIRA, 2012; VAL, 2013). En este marco conceptual, los textos videograbados pueden ser vistos claramente como textos diferidos, dado que están construidos con la finalidad de ser leídos fuera del momento de enunciación. Como ya señalé, eso les confiere características funcionales y estructurales. Asimismo, en tanto objetos físicos, los textos videograbados pueden ser archivados al igual que los escritos.

Si bien existen enormes similitudes entre la escritura y los textos videograbados, lo que hace posible que la videograbación sea una tecnología que permita el desarrollo de una textualidad diferida; también existen importantes diferencias entre los textos diferidos escritos y los textos diferidos registrados y grabados. Estas diferencias son producto, básicamente, del hecho de que la escritura es una tecnología que apunta a representar, gráficamente, las unidades de la lengua, mientras que las audio y videograbaciones apuntan a registrar la lengua.

En un texto escrito, según Sampson (1996), pueden estar representadas las unidades fonológicas, tales como fonemas o sílabas (escrituras fonográficas), o las unidades morfológicas (escrituras logográficas). También hay signos escritos que representan otras unidades, pero son unitarios, no conforman ningún sistema de escritura. En una grabación, por el contrario, se registra la voz, o la imagen o ambos de quien enunció el texto: se registra a los hablantes en el acto del decir.

A nivel cognitivo, el sistema de escritura al ser representacional puede internalizarse y formar parte del propio procesamiento del lenguaje y producir nuevas formas de redescripción representacional (DEFAGÓ, 2012). En sucesivas redescripciones la escritura, en tanto instrumento mediacional de tipo representacional, deja de ser un segundo sistema de señales para pasar a operar de forma directa en el procesamiento del lenguaje, tal como señala Vygotski (2001). Esto supone que el procesamiento del lenguaje en las prác- 
ticas de lectura y escritura no implica un pasaje uno a uno grafema-fonema, sino que el lector/escritor procesa directamente el lenguaje en términos grafémicos, salvo que no conozca la palabra o sea en otro idioma que no domina (BELINCHÓN; RIVIÈRE; IGOA, 1992; NEWMEYER, 1992; DE VEGA; CUETOS, 1999). En el caso de las videograbaciones, en cambio, por ser una tecnología de registro, no existe aún ninguna posibilidad de que esta herramienta pueda internalizarse y pasar a formar parte de la producción del texto. Cuando se está produciendo un texto videograbado, el procesamiento es siempre externo a la herramienta y en eso se aproximaría más a lo que, en relación a ciertas prácticas que se realizan con la escritura, sería una transcripción.

\section{Consolidación de una textualidad diferida en LSU y de una cultura letrada en torno a ésta}

No alcanza con la existencia de una tecnología de la lengua que habilite la textualidad diferida para que la comunidad de hablantes de una lengua se constituya en una comunidad letrada. Se requiere también la existencia de archivos de textualidad diferida, que sean accesibles a un importante sector de la comunidad lingüística que produjo esos textos, y de la especialización de instituciones para su mantenimiento y uso (OLSON, 1996). Asimismo se requiere la generalización, por parte de esa comunidad, del uso de la tecnología y de los textos diferidos en sus prácticas lingüísticas (OLSON, 1996; GOODY; WATT, 1996).

Si bien la LSU carece de escritura y por lo tanto presentó históricamente ciertas dificultades para la consolidación de una cultura letrada, la videograbación es una tecnología que está empezando a ser ampliamente utilizada por la comunidad sorda uruguaya como sustituto del sistema de escritura a los efectos de producir textualidad diferida, ante las necesidades que impone la cultura letrada y globalizada en la que hoy están inmersas las comunidades sordas. 
Con la posibilidad que tienen los sordos en la actualidad de acceso masivo a la videograbación gracias a la tecnología digital (incluso los celulares ya contienen excelentes videocámaras) y de acceso masivo y apropiación de la tecnología de internet, gracias a las políticas públicas de accesibilidad a las tecnologías de la información y la comunicación dirigidas a toda la población del país; los textos videograbados en LSU con las características de textualidad diferida son de uso ya cotidiano por parte de los sordos uruguayos. Pareciera como que, en pocos años, se constituyó una cultura letrada sorda en torno a la tecnología de videograbación, algo que no había, en la comunidad sorda, en torno a la tecnología de la escritura. En ese sentido la tecnología de la videograbación parece una tecnología absolutamente amigable con las características físicas del significante de las lenguas de señas. Tal como se sugiere de los planteos de Val (2014b), una lengua que discurre en el espacio parece que se adapta mejor a una tecnología como las videograbaciones, que permite su registro en todas sus dimensiones, que una tecnología representativa bidimensional como la lengua escrita.

Los sordos uruguayos no parecen en absoluto interesados en las propuestas de escritura que se han hecho para su lengua (signwriting), ni en conocer otras propuestas regionales; mientras que parecen absolutamente adaptados al uso de las videograbaciones en la construcción de textos diferidos. En este sentido es interesantísimo el papel que hoy en día está jugando la videograbación en la comunidad sorda y, da la impresión, que es la tecnología que se va a consolidar como la encargada de sostener toda la textualidad diferida en lengua de señas de aquí en más.

En la actualidad existe una enorme cantidad de videos cortos que circulan por internet, básicamente a través de Facebook y Youtube, en donde los sordos cuentan chistes, realizan cuentos cortos, narran historias, hacen comentarios políticos, realizan cartas, o producen pequeños mensajes. Inclusive el WhatsApp, una nueva modalidad de comunicación por teléfono celular a través de internet, admite el intercambio de videos cortos. Esto ha posibilitado la constitución de un tipo de comunicación en la que se comparte 
el ahora y se intercambian videos cortos en LSU (lo mismo hacen los oyentes con grabaciones de audio). Todos estos tipos de textos videograbados están poblando rápidamente los diferentes sitios por donde discurre la comunidad sorda en internet.

Se puede afirmar, entonces, que en Uruguay los sordos claramente han adoptado la tecnología de videograbación como aquella que permite hacer textos diferidos en LSU. Las tecnologías de videograbación se han hecho realmente accesibles a todos y hoy en día los integrantes de la comunidad sorda uruguaya están equipados con el instrumental correspondiente para realizar todo tipo de videograbaciones, con muy buena resolución. Por parte de la TUILSU y de las escuelas bilingües para sordos (fundamentalmente la de Salto) se han impartido cursos a sordos a efectos de dar a conocer los pasos que deben seguirse para la generación de un texto diferido en LSU de calidad. Asimismo, en el marco de las investigaciones y extensión que se llevan adelante en la TUILSU, se publicó un texto que consigna todas estas especificaciones técnicas a tener en cuenta (VAL, 2013). Esto muestra que se cumple una de las propiedades que debe existir para que se consolide una cultura letrada en torno a una tecnología que habilita la textualidad diferida: el uso masivo de la misma.

A partir de lo señalado anteriormente, en lo que respecta a la consolidación de una cultura letrada en torno a la textualidad diferida, es necesario que también existan archivos de textos diferidos y que estos tengan también un uso masivo por parte de la comunidad. Con la tecnología digital las posibilidades de archivo se facilitan hasta el infinito, dado que cualquier computadora se puede volver un potente archivo y más aún la internet. Sin embargo, para que el archivo tenga funciones dentro de una comunidad letrada, en tanto acervo textual de consulta e intercambio, éste debe ser un archivo público.

En Uruguay, desde el 2012 en adelante, se ha dado un creciente aumento de archivos en internet que apuntan en ese sentido. En estos últimos años, cada vez más instituciones e individuos están caminando en esa dirección con pasos incipientes pero acelerados.

Los Testigos de Jehová es la primera institución que ha especializado su página de archivo en internet para contener textos diferi- 
dos en lenguas de señas de todas partes del mundo, la que ya tiene décadas de funcionamiento ${ }^{1}$. Los textos de los Testigos de Jehová en LSU son de aparición más reciente (a partir del año 2013) ${ }^{2}$.

Otras instituciones en Uruguay están dando similares pasos. En la Asociación de Sordos existe el archivo de videos de historia de vida de los sordos viejos, pero no es de fácil acceso público y está en soporte analógico. Sin embargo, la institución publica en internet videos en su página de Facebook y en su canal de Youtube $^{3}$. Las Asociaciones de Sordos de la ciudad de Salto y de la ciudad de Maldonado también publican videos en su Facebook ${ }^{4}$.

En las escuelas bilingües para sordos donde se trabaja esta textualidad ya se han empezado a guardar textos videograbados con fines públicos e inclusive se impulsan blogs institucionales en los que se archivan esos videos ${ }^{5}$.

Lo mismo ocurre con la TUILSU/Universidad de la República, que también ha realizado tímidos movimientos en ese sentido. En el año 2011 la TUILSU sacó un DVD con las actas de un congreso que realizó en Montevideo en el que los sordos publicaron sus resúmenes en textualidad videograbada en LSU (FOJO y PELUSO, 2011). En el 2013 se promovió la elaboración de artículos académicos en LSU videograbada, para los docentes sordos, que aún no fueron publicados y se proyecta la publicación y archivo de un importante acervo textual en LSU a construir (tanto a través de realización de textos nuevos, como de traducción de textos que están actualmente en español escrito). Cada vez más la TUILSU viene elaborando material videograbado, lo que incluye Web-series e informativo ${ }^{6}$.

Asimismo, paulatinamente se comienza a ver el interés, por parte de algunos sordos, sin representar ninguna institución, de archivar sus textos (cuentos, chistes, relatos históricos, etcétera) en sus páginas de Facebook o en canales de Youtube con la intención de que sean accesibles para muchos integrantes de la comunidad ${ }^{7}$.

Tanto los canales de Youtube como las páginas de Facebook son abiertas con la intención de archivar y difundir los textos diferidos que se realizaron. Sin embargo, también existen formas de archivo a través de los Facebook personales de todos los integrantes de la 
comunidad sorda. En ese caso son de destacar los Facebook personales de los sordos citados así como el de Elaine Fernández ${ }^{8}$.

Si bien estos sitios son personales, por las características de la internet y de la posibilidad de intercambio que esta trae, así como por la propia intención de quienes los llevan adelante, los mismos se han vuelto claramente de dominio público en el contexto de la comunidad sorda uruguaya.

\section{Traducir e interpretar en el nuevo contexto tecnológico}

En este nuevo contexto tecnológico, en el que las videograbaciones se han consolidado como una tecnología clave para la realización de textualidad diferida en LSU, se abre, para el ejercicio de la profesión de traductor en el campo interlingüístico de las lenguas orales y de señas, dos contextos claramente diferenciados. Por un lado el trabajo con textos orales, evanescentes, que son parte de la comunicación entre hablantes de diferentes lenguas y por otro lado el trabajo con textos diferidos, en los que se puede actualizar una estructura de demora que habilita reflexión, control y planificación sobre todo el proceso.

Como plantea Famularo (2011) las prácticas de traspaso de una lengua a otra pueden ser llamadas, en términos generales, prácticas traductológicas, dado que involucran en todos los casos procesos cognitivos y lingüísticos similares: aquellos que suponen un hablante con dominio de dos sistemas lingüísticos en la situación de hacer transparente, en una lengua, un texto producido en otra, así sea este oral o escrito. Sin embargo, resulta obvio que los procesos cognitivos, lingüísticos y situacionales que están implicados son completamente diferentes en los casos en los que quien se encarga de transparentar textos interlingüisticamente está trabajando con hablantes en contextos de oralidad (relaciones cara a cara), que en los casos en los que se está trabajando con textos diferidos (escritos y videograbados).

En los procesos de interpretación, el profesional está en el marco de un contexto de oralidad. Esto supone que se recibe el texto, 
que es evanescente, en una lengua, y se lo tiene que traspasar a la otra de forma simultánea. Esto hace que tenga poco o nulo margen para pensar sobre los textos y las lenguas implicadas. En estas situaciones no hay tiempo de generar esa estructura de demora que habilita la reflexión metalingüística, la planificación y el control.

En los procesos de traducción, el traductor se enfrenta a un texto diferido, que puede ser un texto escrito en español o un texto videograbado en lengua de señas, y a punto de partida de dicho texto se realiza otro texto, también diferido, en la otra lengua. Este proceso de trabajo con textos diferidos, lejos de la premura y la exigencia que conlleva la interpretación en la oralidad, permite la constitución de una estructura de demora en la que se habilita el control, la planificación y toda la actividad metalingüística y metacognitiva que se pone en juego cuando se procesan este tipo de textos. Por otra parte, mientras que el texto que se produce en los contextos de interpretación es tan evanescente como el texto de partida (salvo que se esté registrando la situación) y debe ser tenido en cuenta como tal; los textos traducidos, en la medida en que son diferidos, son realizados para permanecer. La traducción necesariamente genera textos permanentes, por lo tanto debe mantener ciertas estructuras que también hacen a su estética. Esta propiedad de permanencia está en la base constitutiva de la propia producción del texto traducido.

Teniendo en cuenta estas nuevas posibilidades traductológicas que se abren con la tecnología de las videograbaciones, se puede afirmar que los campos de la traducción LSU-español se han vuelto diversos y absolutamente necesarios para una comunidad sorda que cada vez más necesita y exige textualidad diferida en su propia lengua. Estos nuevos campos son: la traducción de un texto escrito en español a un texto videograbado en LSU; la traducción de un texto videograbado en LSU a un texto escrito en español; la subtitulación en LSU de una película hablada en español; y la subtitulación mediante español escrito y audiodescripción de una película hablada en lengua oral o en lengua de señas.

La traducción de un texto escrito en español hacia un texto videograbado en LSU supone la deconstrucción de la estructura sin- 
táctica del texto en español para poder ser reconstruida en la LSU videograbada. Este tipo de traducción ha representado un sinnúmero de interrogantes que aún no tienen respuesta en el estado actual de las investigaciones y prácticas en el campo.

La mayor interrogante surge porque el español y la LSU no son paralelas en cuanto a los procesos de intelectualización y de gramatización que se han aplicado sobre ellas. Debido a esto, son dos lenguas que, por un lado, tienen diferentes desarrollos de los campos léxicos relacionados con el quehacer científico, siendo que la LSU, al no tener hasta el momento comunidades científicas de sordos en determinadas áreas, no se vio necesitada de desarrollar los campos léxicos vinculados a éstas. Por otro lado, son dos lenguas que presentan diferente desarrollo de la sintaxis y de géneros discursivos, entendidos estos como las diferentes maneras de elaborar tipos relativamente estables de enunciados, permitidos por cada esfera de uso de la lengua. Mientras que el español ha especializado sintaxis y géneros discursivos para la escritura formal; en la LSU esta especialización está en proceso dado que hasta el momento era una lengua que carecía de textualidad diferida. Tal como fue señalado por Lodi (2014), similares procesos están ocurriendo en otras lenguas de señas de la región.

En relación a esta situación diferencial entre las lenguas se presentan tres escenarios que son un desafío a la hora de pensar las metodologías traductológicas: ¿Qué se hace cuando en el texto en español aparece léxico que no existe en la LSU? ¿Qué se hace cuando en el texto en español aparece léxico que si bien existe en la LSU, por las características del desarrollo sociohistórico de la comunidad, solo podría ser entendido por una elite? ¿Se debe respetar la organización textual que propone el texto en español?

Por su parte, la traducción de un texto videograbado en LSU hacia un texto escrito en español tiene menos posibilidades de ocurrir, dado que mientras del español escrito a la LSU videograbada hay una biblioteca casi infinita de textos a traducir; de la LSU videograbada al español todavía existen muy pocos textos a traducir, en el estado actual de la comunidad sorda uruguaya. Sin embargo, 
este tipo de proceso traductológico es claramente un desafío creciente frente a la necesidad, por ejemplo, que tienen los sordos en la academia de poder escribir el texto en su propia lengua mediante una videograbación para luego solicitar sea traducido a la lengua oral escrita en la que quieren realizar una publicación (similar situación ocurre a muchos académicos hispanohablantes cuando nos enfrentamos a una publicación en portugués o en inglés).

Otro campo traductológico es la subtitulación en LSU de una película hablada en español. Esto supone la inclusión de un recuadro pequeño en el que aparecen los subtítulos videograbados en LSU. En general aparecen diferentes traductores según cada personaje de la película. Este tipo de subtitulado se considera una traducción porque se trabaja con textualidad diferida. En este momento existen cinco películas uruguayas, tres de adultos (Viaje hacia el mar, El círculo y Corazón de fuego) y dos infantiles (Selkirk, el verdadero Robinson Crusoe y ANINA), traducidas bajo esta modalidad.

Por último tenemos la subtitulación en español escrito y audiodescripción de una película hablada en español o de una película hablada en LSU. En ambos casos en TUILSU se siguen similares reglas, que son una versión adaptada de la norma española UNE153010: 2003. Fundamentalmente se pone en diferente color según sea el personaje que habla, se adecua el largo medio de los enunciados para respetar el ritmo de la película y, en caso de haber sonido, se lo describe a efectos de que los sordos puedan tener también ese dato, que muchas veces puede ser central en una escena (AEN/CTN, 2003; TANCREDI, 2013).

En este sentido fue particularmente interesante el proceso seguido en la subtitulación de la Serie Identidades realizado en la TUILSU (ÁLVAREZ; MUSLERA; RODINO, 2014). Esta Serie tiene como característica el haber sido filmada en ausencia de sonido, eso formó parte del proyecto inicial de trabajo, y el estar casi por completo hablada en LSU (VAL, 2014a; PELUSO, 2014). Igualmente, al no tener sonido, las pocas partes que son habladas en español tampoco se escuchan. En el momento de subtitularla al es- 
pañol escrito, el equipo que trabajó en ello, conformado por sordos y oyentes, se enfrentó a la interrogante acerca de hasta qué punto el subtitulado en español debía respetar la estructura de la LSU. Esta interrogante no solo es técnica, sino también política (ÁLVAREZ; MUSLERA; RODINO, 2014). Los sordos, acostumbrados a defender su lengua de las interferencias del español, y a promover prácticas traductológicas hacia la LSU, querían que el español del subtitulado reflejara las estructuras de la LSU, aún a costa de que aparecieran estructuras raras en español. Pienso que esto muestra, a nivel simbólico, cómo juegan las relaciones políticas entre las lenguas, las que se instituyen al momento de establecer posturas metodológicas, pero también éticas, frente a la traducción en el contexto interlingüístico actual: el español es visto como la lengua de colonización, poderosa, por lo que se puede interferir sobre ella sin que esto sea amenazante para la misma; en cambio la LSU es vista como la lengua que hay que defender frente a las interferencias del español. Este tipo de traducción, a mi criterio, pone sobre la mesa estas cuestiones y ayuda a recolocar a las lenguas y a sus hablantes en planos de igualdad. Asimismo, esta traducción, mostró indicios de un cambio en la matriz traductológica hegemónica que señala que son los valorados productos textuales generados en español (escritos o filmados) los que deben ser traducidos a la LSU. A diferencia de dicho presupuesto, esta traducción nos enfrenta a un producto fílmico valioso, generado en LSU, que debe ser traducido para que quienes no hablan dicha lengua (la mayoría) puedan acceder al mismo.

\section{Acerca de las metodologías de traducción}

En la TUILSU estamos desarrollando una metodología de traducción del español escrito hacia la LSU videograbada tomando en cuenta los avances en la metodología de grabación por fragmentos-párrafos (que consiste en ir grabando fragmentos, que funcionan como párrafos que luego se unen) y los trabajos realizados por el 
equipo de traducción de los Testigos de Jehová, quienes son los únicos que tienen, en la actualidad, un importante camino recorrido en el campo de la traducción de textos escritos en lengua oral a videograbaciones en lengua de señas (TESTIGOS DE JEHOVÁ, 2012; SÁNCHEZ; SALGUERO, 2011; GÓMEZ; SALGUERO, 2013). Como los Testigos de Jehová traducen fundamentalmente textos religiosos, el desafío del equipo de trabajo TUILSU es elaborar una metodología que tenga en cuenta las particulares necesidades que tienen los textos académicos.

Nosotros nos basamos en el modelo de trabajo que proponía el equipo de traducción de los Testigos de Jehová de Buenos Aires en el año 2012, que tenía ya un fuerte desarrollo en la región (TESTIGOS DE JEHOVÁ, 2012).

En lo relativo a los equipos de trabajo, ellos sostienen que éstos debe estar formados por tres personas con rol definido: Traductor, Revisor y Corrector; ser interlingüísticos e interculturales y estar constituidos por sordos y oyentes.

Las funciones para cada rol en el inicio de la traducción son: el Traductor, que puede ser un oyente o un sordo con un gran dominio del español escrito, hace un mapa conceptual (hasta el año 2010 hacía glosas o ambos) proponiendo una traducción del texto de partida a la lengua de señas; el Revisor, que puede ser oyente o un sordo con fuerte conocimiento del español escrito, estudia si lo que realiza el Traductor es fiel al texto de partida en español; el Corrector, que tiene que ser sordo con un fuerte conocimiento de LSU, corrige las el mapa conceptual y estudia que la traducción sea natural. Se trabaja con la idea de que los tres participen de la traducción al inicio haciendo en conjunto o por separado la traducción inicial en mapas conceptuales. Igualmente se mantienen los roles y es el traductor que toma la iniciativa en el proceso.

Luego de conformado el equipo, la traducción sigue varios pasos sucesivos, cuyo orden se respeta de forma estricta.

El paso número 1 es la Preparación. El equipo lee y analiza el texto por separado, según una plantilla predefinida que permite 
identificar el marcrotexto, los paratextos, así como también indica la realización de una síntesis del texto (se busca fundamentalmente establecer el propósito del texto y sus conclusiones). En la misma plantilla se identifican: tema, propósito, público objetivo, tono y estructura.

En esta etapa cada miembro del equipo comienza a realizar glosas posibles de traducción y mapas de ideas.

El paso número 2 es la Reunión del proyecto. La meta de la reunión es llegar a un acuerdo del significado del texto fuente, luego que cada uno hizo la plantilla. Se trata de aceptar el criterio de la mayoría.

Se hace el mapa de ideas entre todos, que luego va a regir la traducción al video. Esto es un momento clave del proceso de traducción porque es cuando se despoja totalmente, al texto fuente, de su estructura superficial y queda exclusivamente la estructura profunda. Al desaparecer la estructura del español, se evita así toda interferencia de éste sobre la lengua de señas.

El paso número 3 es la Traducción inicial que apunta a generar el primer video borrador. A partir del mapa de ideas elaborado en la etapa anterior, se hace el primer video. Este video es un borrador, una traducción rápida. No se busca lograr un video perfecto, sino una primera versión en lengua de señas del texto de partida. A partir de ese video se detectan los problemas, tanto en el proceso como en el producto.

El primer video lo realiza el traductor, que en un inicio en general era oyente, pero que ahora es preferentemente sordo con comprensión del español. El traductor sordo se apoya en la interpretación del texto escrito que hace otro miembro oyente del equipo.

Ese primer texto lo revisa el sordo y plantea todas sus críticas e indica los cambios a realizar. A partir de esto se vuelve a filmar. Este segundo video lo realiza el sordo y se descarta la primera traducción, y así sucesivamente hasta alcanzar un video borrador que se entienda como de buena calidad. La idea es que este proceso sea rápido para que no se pierdan las ideas. 
El paso número 4 es la Revisión de la traducción. A partir de este primer borrador se chequea que lo grabado respete el mapa de ideas: no puede haber pérdida ni agregados. Quien controla esto es el revisor. Acá ya no aparece más el texto fuente, dado que se chequea a punto de partida del mapa de ideas. Para el equipo de traducción de los Testigos de Jehová esto es clave, porque es lo que permite despojarse de la forma del texto fuente y se reducen los riesgos de realizar español señado (se busca lo que se ha denominado una traducción idiomática).

Cuando se encuentran errores se vuelve a grabar el párrafo de vuelta. Se trabaja con grabación por párrafos y, en esta etapa, el borrador es estudiado párrafo por párrafo.

El paso número 5 es la Verificación y finalización. En esta nueva etapa se estudia el texto en su globalidad, ya no párrafo por párrafo. Se chequea cohesión y coherencia general del texto y se chequea, con el mapa de ideas, que la traducción sea fiel al texto de partida en términos más globales.

El paso número 6 es la Grabación del video borrador final. Luego de pasar por los chequeos de las etapas 4 y 5 y se tiene la certeza de que la traducción es la correcta, se vuelve a grabar, en este caso el video borrador final. A veces ya en la etapa 3 quedó realizado el video borrador, pero a veces, luego de las revisiones, hay que seguir corrigiendo para incorporar las correcciones que se le realizaron en las siguientes etapas.

El paso número 7 es la Revisión externa. Se constituye un panel revisor externo conformado por sordos. Se les pide que lo vean y a partir de allí se les hacen preguntas sobre el texto. Se filma a los sordos haciendo sus comentarios y sugerencias a efectos de que sus opiniones queden grabadas y puedan ser posteriormente analizadas por el equipo de traducción. En caso necesario, se vuelve a grabar incorporando esas ideas y sugerencias; pero solo se regraban los párrafos cuestionados.

El paso número 8 es la Grabación del video apuntador. A partir del video borrador se graba el video apuntador. El video apuntador 
es la versión final y previa a la traducción. Siempre se utiliza la metodología de la grabación por fragmento-párrafo.

El paso número 9 es la Marcación de la ubicación de los OST. El equipo señala dónde van esas marcas, ejemplo, párrafo 1, salmo 3, y así sucesivamente.

El paso número 10 es la Grabación final en estudio. El texto final se graba por párrafos, con inicio y final fundido a negro. En relación a las tomas, cada fragmento-párrafo no dura más de dos minutos y medio. Para la grabación se utiliza el video apuntador. El hablante que va a ser videograbado queda frente a la cámara y es sostenido por el video apuntador que se proyecta simultáneamente en una serie de monitores dispuestos en círculos para que quien está siendo filmado no los pierda nunca de vista. Si se usara un único monitor, el hablante no podría ir cambiando su mirada en el espacio señante (lo que es discursiva y sintácticamente relevante en lengua de señas) y quedaría estático siempre mirando hacia adelante. Asimismo, está el equipo traductor acompañando el proceso.

El fondo es blanco y se lo alumbra con un color determinado para cambiar el color según se quiera por el tipo de texto que se está traduciendo.

El paso número 11 es el Archivo de la traducción. Todas las traducciones se archivan junto con el diario de trabajo que se realiza a lo largo de las mismas, en el que se describe el proceso y se plantean los desafíos y problemas que surgieron durante el mismo.

Este modelo de traducción es el primero que se desarrolla con este nivel de exhaustividad metodológica para la traducción de textos diferidos de las lenguas orales hacia las lenguas de señas. Con esta metodología se han generado, a nivel internacional, una enorme cantidad de traducción de textos religiosos, lo que incluye varios libros de la Biblia. Ya ha sido traducido enteramente el llamado Nuevo Testamento en ASL y varios libros del Antiguo Testamento. Brasil, Colombia, Corea, Italia, Rusia, entre otros, están traduciendo el Nuevo Testamento.

En la TUILSU este modelo traductológico aún no se ha podido incorporar totalmente. El aprendizaje de la técnica de los mapas 
conceptuales no es tarea sencilla y el uso del sistema de monitores como video apuntador no resultó una tecnología amigable para quienes cumplen el rol de ser los hablantes a videograbar. La modalidad que nos está resultando más manejable, por el momento, es el trabajo de cada párrafo con el sordo que va a videograbar el texto y su siguiente grabación apelando a la memoria inmediata del hablante. Por eso la definición de párrafo a realizar en el video se basa en dos factores: identidad temática y cantidad de información a ser memorizada con comodidad. Por lo tanto, los párrafos en LSU videograbada no coinciden, necesariamente, con los que aparecen en el texto de español.

La traducción de la LSU al español es un desafío diferente. No existen escuelas traductológicas ya consolidadas para este tipo de práctica, por lo que se deben ir resolviendo los problemas sobre la marcha. Hasta ahora hemos traducido la primera temporada de la Serie Identidades (ÁLVAREZ; MUSLERA; RODINO, 2014) y algunos textos elaborados por los profesores sordos de la TUILSU en LSU para su presentación ante congresos o para ser publicados (cf. Nota de los traductores en DE LEÓN, FLORES, GONZÁLEZ, ROMERO y TOURÓN, 2014). En este caso se utiliza similar procedimiento traductológico que el que se realiza para cualquier traducción entre dos textos escritos en lenguas orales.

\section{Conclusiones}

A lo largo de este artículo he presentado y articulado los principales hallazgos que se vienen realizando en el marco de investigaciones que tienen, por un lado, el objetivo de indagar acerca de las características y condiciones de la textualidad diferida en LSU y, por otro lado, las posibilidades teóricas y metodológicas que esto trae para el desarrollo de prácticas traductológicas.

Creo firmemente que en el estado actual de la comunidad sorda y de su relación con las prácticas letradas en español escrito y en LSU videograbada, es necesario comenzar, con urgencia, a pro- 
ducir una textualidad diferida en LSU que acompañe las nuevas funciones que ocupa dicha lengua con su ingreso a los ámbitos formales. Si bien en la actualidad los sordos lograron que su lengua ingresara en las tres ramas de la educación pública del Uruguay (Primaria, Secundaria y Universidad); no ingresó, concomitantemente, a dichos espacios una textualidad diferida en dicha lengua, por lo que éstos deben estudiar exclusivamente en español escrito.

Esto indica que los sordos requieren que sea traducida toda la biblioteca mundial; lo que es un actividad que, obviamente, no tiene fin pero que requiere de un comienzo.

La presencia de una fuerte escuela traductológica, no solo acompañaría los naturales procesos de generación de textualidad diferida por los que espontáneamente la comunidad sorda ya se ha encaminado, sino que redundaría en un cambio en las relaciones de poder entre las lenguas y, por supuesto, en las propias estructuras de la LSU. La tecnologización de una lengua trae cambios profundos en ella y en sus hablantes (ONG, 1982; GOODY; WATT, 1996; AUROUX, 1992). Por ejemplo, siempre se ha dicho que el género policial en la lengua francesa surgió como efecto de la traducción, desde el inglés, de las novelas policiales que se desarrollaron en esta lengua. Por lo tanto, es de esperar que con una fuerte escuela traductológica ocurra la especialización del decir en nuevos géneros discursivos por los que aún la lengua de señas no ha transitado (LODI, 2014).

En ese sentido, las prácticas de traducción de una lengua a otra son siempre parte de políticas lingüísticas y, generalmente, tienen sus efectos en ambas lenguas. Por eso deben ser también vigiladas políticamente, por las consecuencias que esto puede acarrear en términos de las relaciones entre comunidades y hablantes, así como en términos de las propias estructuras lingüísticas y enunciativas de las lenguas involucradas.

A su vez este presupuesto de que las videograbaciones en LSU son textualidad diferida y de que es posible la traducción desde el español escrito a la LSU videograbada y viceversa, tuvo como efecto práctico un cambio en el plan de estudios de la TUILSU. Como se plantea en el propio Plan de estudios de la tecnicatura en 
interpretación y traducción LSU - español (TUILSU, 2014, s/p) el perfil del egresado supone que:

Los traductores sordos y oyentes estarán habilitados a participar en equipos en los que se trabaja en dupla sordo-oyente con textualidad diferida y en los que realizarán las siguientes actividades:

1. Realizar traducciones de textos escritos en español hacia videograbaciones en LSU y viceversa.

2. Realizar subtitulación para sordos mediante español escrito (clause caption), de cualquier producto audiovisual, tanto producido en español como en LSU o ambos.

3. Realizar subtitulación a través de videograbaciones en LSU de los productos audiovisuales en español (esta técnica de subtitulación consiste en sustituir el español escrito del tradicional subtitulado por cuadros con videos en LSU en los que se traduce a dicha lengua lo que se dice en español).

Con este cambio de plan se apunta en dos direcciones. Por un lado al ingreso de estudiantes sordos a la carrera para formarse como traductores, lo que abre un espacio que antes tenían vedado dado que la formación era para intérpretes oyentes. Por otro lado apunta al desarrollo de una escuela traductológica que pueda hacer frente a las nuevas exigencias de una comunidad sorda que está ocupando nuevos espacios sociales. Se recoge así una necesidad imperiosa de la comunidad, que cada vez más exige la presencia de traductores calificados.

\section{Notas}

1. Sitio Web: Testigos de Jehová < http://www.jw.org > 
2. <http://www.jw.org/ugy/publicaciones/>

3. Facebook: ASUR, Asociación de Sordos del Uruguay < https://www.facebook.com/asur1928? fref =ts $>$ Youtube, ASUR Uruguay $<$ https://www.youtube.com/user/asur1928>

4. Facebook: ASS, Asociación De Sordos Salto: <https://www.facebook.com/ asociacionde.sordossalto.1/videos $>$ Facebook: A.SO.MA, Asociación de Sordos de Maldonado:<https://www.facebook.com/pages/Asociaci\%C3\%B3n-de-Sordos-de-Maldonado $/ 1560107274213102$ ?fref $=$ photo\&sk = videos $>$

5. Blog: Escuela 116 bilingüe de sordos de Salto:<https://www.google.com/ search?rls $=$ aso $\&$ client $=$ gmail $\& q=$ escueladesordosdesalto $>$ Youtube: escuela 116 espacio sordos: $<$ https://www.youtube.com/results?search_query $=$ e scuela $+116+$ espacio + sordos $>$ Blog: Escuela 197 bilingüe para sordos de Montevideo: < http://www.eduescuela197.org/cuento--oliver-button-es-una-nena.html > Blog: Escuela 84 bilingüe para sordos de Maldonado:<http://sordos84.blogspot.com/>

6. Youtube: TUILSU FHCE<https://www.youtube.com/channel/UCk21QJLeZ1s8z1QxHf0eEhw $>$. Grupo público de Facebook: TUILSU (FHCE, Udelar)<https://www.facebook.com/groups $/ 592740534120788 /$ ? fref $=$ ts. Youtube: TUILSU informativo: <https://www.youtube.com/channel/UCERu9HBFZm0K1bQHYeV3NFQ>

7. Youtube: canal DosEme Vlog de Maximiliano Meliande<https://www.facebook.com/DosEmeVIDEO?pnref=story. Facebook: Dos Eme-Maximiliano Meliande, Actor/Director <https://www.facebook.com/DosEmeVIDEO. Youtube: canal de Carina Romero Bacigalupo <https://www.youtube.com/channel/ UCZB3EUM_LmfKYCwA_Fhv_ww/featured. Facebook: Carina Romero Bacigalupo, Taller/Estudio < https://www.facebook.com/pages/Carina-Romero-Baci galupo $/ 452369381610832 ? \mathrm{fref}=\mathrm{ts}>$

8. Facebook: Elaine Fernández $<$ https://www.facebook.com/efff68?fref $=$ ts $>$ 


\section{Referencias}

AEN/CTN. Norma española UNE 153010I. Madrid: AENOR, 2003

ÁLVAREZ, C.; MUSLERA, S. y RODINO, M.E. Experiencia de traducción de la Serie Identidades. En: TUILSU-imagen producciones 2014: Consideraciones teóricas, metodológicas y políticas. Montevideo: TUILSU/UdelaR, 2014, pp. 25-30.

AUROUX, S. A revolução tecnológica da gramatização. 1. ed. Campinas: Editora da Unicamp, 1992.

BELINCHÓN, M., RIVIERE, A. e IGOA, J.M. Psicología del lenguaje. Investigación y teoría. Madrid: Ed. Trotta, 1992..

DE LEÓN, A. FLORES, I. GONZÁLEZ, R. ROMERO, C, y TOURÓN, G. Los sordos, el español escrito y la comunicación. En: TUILSU-imagen producciones 2014: Consideraciones teóricas, metodológicas y políticas. Montevideo: TUILSU/UdelaR, 2014, pp. 31-35.

DE VEGA, M. Y CUETOS, F. (Coord.) Psicolingüística del español. Madrid: Editorial Trotta, 1999.

DEFAGÓ, C. Tras los rastros del lenguaje: revisión teórica del modelo RR de Karmiloff-Smith. En: Revista Argentina de Ciencias del Comportamiento (RCCA). Vol, 4 Nro 1, 2012, pp. 44-55.

FAMULARO, R. Despejar X. Interpretación en Lengua de Señas y en Lengua Oral. Montevideo: TUILSU/UdelaR, 2011.

FOJO, A. y PELUSO, L. (Org.) Actas del II Encuentro Internacional de Intérpretes y I Encuentro Regional de Sordos y I Congreso Regional de Investigadores de Lenguas de señas y Culturas Sordas. Montevideo: TUILSU/UdelaR, 2012.

GÓMEZ, P. J. y SALGUERO, E. El intérprete de LS en el campo religioso. Trabajo presentado en el III Encuentro Latinoamericano de Sordos e Intérpretes de Lenguas de Señas. Paraná, junio de 2013. 
GOODY, J. y WATT, I. Las consecuencias de la cultura escrita. En: GOODY, J. (Comp.) Cultura escrita en sociedades tradicionales. Barcelona: Gedisa, 1996, pp. 39-82.

LARRINAGA, J.A. y PELUSO, L. Sordera, escritura y enseñanza de lengua. En: Memorias de las XVI Jornadas de Investigación y Quinto Encuentro de Investigadores de Psicología del Mercosur, Tomo II. Buenos Aires: Ediciones de la Fac. de Psicología de la UBA, 2009, pp.345-347.

LODI, A.C.B. Texto e discurso em Libras: possibilidades de apropriação de práticas de leitura e produção de textos/discursos por alunos surdos. En: ERNST, A.; LEFFA, V.J.; SOBRAL, A. (Org.) Ensino e Linguagem. Novos Desafios. Pelotas: EDUCAT, 2014, pp.97-126.

NEWMEYER, F. (Comp.) Panorámica de la lingüística moderna de la Universdiad de Cambridge. III. El lenguaje: Aspectos psicológicos y biológicos. Madrid: Visor, 1992.

OCHS, E. Planned and unplanned discourse. En: GIVON, T. (Ed.) Discourse and Syntax. New York: Academic Press, 1979, pp. 51-80.

OLSON, D.R. La cultura escrita como actividad metalingüística. En: OLSON, D. R. y TORRANCE, N. (Comps.) Cultura escrita y oralidad. Barcelona: Gedisa, 1996, pp. 333-358.

ONG, W. Orality and Literacy: The Technologizing of the Word. 1. ed. London: Methuen, 1982.

PELUSO, L. Estudios interculturales y cultura escrita: algunas problematizaciones en torno al concepto de escritura y de sujeto letrado. En: Sociedad de Dislexia del Uruguay. Entre el sueño y la realidad: nuestra América Latina Alfabetizada. Montevideo; Edición de la Sociedad de Dislexia del Uruguay, 2007, pp. 313-320.

PELUSO, L. Serie Identidades: Consideraciones conceptuales y metodológicas. En: TUILSU-imagen producciones 2014: Consideraciones teóricas, metodológicas y políticas. Montevideo: TUILSU/UdelaR, 2014, pp. 7-15. 
PELUSO, L. y SILVEIRA, M. Chat y videos en LSU: uso de las XO en la educación bilingüe de sordos. Trabajo presentado en 1.edu Apropiación y Desarrollo: Modelos 1 a 1, Montevideo: Flor de Ceibo/UdelaR, 2012.

SÁNCHEZ, O. y SALGUERO, E. El intérprete de LS en el campo religioso: desafíos y logros. En: Actas del II Encuentro Internacional de Intérpretes y I Encuentro Regional de Sordos y I Congreso Regional de Investigadores de Lenguas de señas y Culturas Sordas. Montevideo: TUILSU/UdelaR, CD-rom, 2011.

SAMPSON, G. Sistemas de escritura. 1. ed. Barcelona: Gedisa, 1996.

TANCREDI, M. El subtitulado para sordos, tarea del interprete de lengua de señas. Trabajo presentado en el III Encuentro Latinoamericano de Sordos e Intérpretes de Lenguas de Señas. Paraná, junio de 2013.

TESTIGOS DE JEHOVÁ. Curso sobre metodología de traducción español-LSA. Buenos Aires, julio 2012.

TUILSU. Plan de estudios de la tecnicatura en interpretación y traducción LSU español. Montevideo: Facultad de Humanidades y Ciencias de la Educación, 2014.

VAL, S. Videograbación de hablantes de lengua de señas: algunos elementos a considerar. Montevideo: TUILSU, 2013.

VAL, S. Consideraciones acerca de la realización de la Serie "Identidades". En: TUILSU-imagen producciones 2014a: Consideraciones teóricas, metodológicas y políticas. Montevideo: TUILSU/UdelaR, 2014, pp. 17-24.

VAL, S. Lenguas de Señas: dimensiones e iconicidad. Esbozo para una investigación basada en la teoría cinematográfica. En: Lengua de Señas e Interpretación, $\mathrm{N}^{\circ}$ 5, 2014b, pp. 129-147.

VYGOTSKI, L. Pensamiento y lenguaje. En: Obras escogidas. Tomo II., Madrid: Visor, pp. 9-348, 2001.

Recebido em: 29/06/2015

Aceito em: 08/09/2015 